\title{
Focus on Methodology: A sense of sociomaterialism: How sociomaterial perspectives might illuminate health professions education
}

\author{
S. Burm \& A. MacLeod
}

\begin{abstract}
Emerging from the field of socio-technical studies (STS), "sociomaterial" refers to a diverse group of theoretical approaches that, broadly speaking, share a commitment to symmetrically privileging both social and material elements. Not surprisingly, the ways in which a sociomaterial researcher engages in the process of collecting and analysing data will differ from studies that foreground a more human-centered point of view.

This paper introduces researchers to the principles informing sociomaterial research and demonstrates their application in the context of a problem that has reached rampant levels among healthcare professionals — burnout. In this paper, we provide an accessible, in-depth description of what we mean by the sociomaterial, describe its historical roots and elucidate what it means to engage in empirical sociomaterial work. In so doing, our goal is to illuminate the potential of sociomaterial studies for exploring the complexity of health professions education.
\end{abstract}

Keywords: sociomateriality; materiality; ethnography; health professions education

Continuing Professional Development \& Medical Education, Faculty of Medicine, Dalhousie University

Correspondence

Anna MacLeod

Continuing Professional Development \& Medical Education

Faculty of Medicine

Dalhousie University

Halifax, Nova Scotia

Canada

Tel: +19024947861

Email: anna.macleod@dal.ca 


\section{Introduction}

What comes to mind if we ask you to imagine a "typical" health professions education (HPE) scenario? Perhaps you pictured a lecture theatre, furnished with stadium-style seating, a podium at the front of the room and a large screen behind a single professor delivering an engaging lecture. Maybe you imagined a simulation suite, with a pair of students trying their best to maintain a sterile field while prepping for a practice procedure on a manikin. Maybe you envisioned a busy ward, with students quietly "shadowing" experienced clinicians as they work with patients.

These vignettes, along with countless others, are all part of the complex experiences of HPE - a very person-focused endeavour. We describe our professional work in terms like "patient centred", and we use words like "student centred" to describe our educational philosophies. In each of the scenarios described above, we almost certainly foreground the people in our imaginings. The professor at the podium, the students in the simulation suite, the clinical teacher, learners and patients on the ward-our work as health professionals and as educators of health professionals is surely a human endeavour. However, the people of HPE do not exist on their own, in a vacuum. Rather, they are part of a vast and ever-evolving network of elements that includes not only people but also non-people, or things. And while it may be easy to consider the non-human actors in these scenarios playing only a small, supporting role, on closer inspection, this might not actually be the case.

Take, for example, the pair of students working in a simulation suite. The materials-in other words, the physical things - in the space are worth considering in terms of their contribution to student learning. The manikin, for example, is a fascinating material. Its vacant expression, rubber-like skin, physical shape and size all have an influence on the educational experience. And while the manikin is an obvious influencer, likewise are a whole host of other material factors, including the height of the bed, the smoothness of the wheels on the tray, the proximity of the rubbish bin from the bed and an innumerable number of other factors. Attempting to truly understand the complexity of the simulation scenario without paying attention to these other contributing factors allows for only a limited, or skewed, perspective.

We believe that centering the material complexity of HPE—-deliberately paying attention to non-humans-may actually help us to better understand the human experience of health professions education. In the paper that follows, we will provide a more in-depth description of what we mean by sociomaterial, describe its historical roots and provide more information about what it means to engage in empirical sociomaterial work.

\section{What do we mean by sociomaterial?}

Let's begin by simply breaking down the term into its two key components: social and material. With respect to "social", we mean everything that is, broadly speaking, related to the organisation of society. These are, generally speaking, human concerns and include, for example, issues that characterise HPE, and the world, more broadly, such as gender, relationships, social class and the rules and norms that guide them. One 
point of clarification here, raised by Hassan (2016), is that human and social are terms that should perhaps not be used interchangeably. He noted that the material human body can demonstrate agencies of its own that are separate from inscribed social human elements.

What do we mean by "material"? Materiality encompasses physical things that have spatial characteristics, including a specific shape, location, volume and mass (Faulkner $\&$ Runde, 2010; Hassan, 2016; Kallinikos, 2012). Leonardi (2012) added a caveat to the definition, describing technology as a distinct type of material, which combines a physical, material form with digital matters in such a way that the technology can exist in particular configurations that endure across time and space.

Those who operate from a sociomaterial perspective believe that both social and material elements are working together to produce HPE. This means that we strive to deliberately account for both social and, in particular, material elements in our scholarship. The rationale for this "symmetry" is based in the historical origins of sociomateriality.

\section{What are the origins of sociomaterialism?}

The work that we do in organisations-whether those be institutions of HPE or others-is inherently tied up with materiality (Orlikowski \& Scott, 2008). Neglecting to attune to the material may, at the very least, limit the result of our research if not misrepresent its complexity. Yet, for the most part, researchers in the realm of HPE have not deliberately turned a critical eye to the material elements of our educational processes (Fenwick, 2014). As Barad (2003) famously noted, "Language matters. Discourse matters. Culture matters. But there is an important sense in which the only thing that does not seem to matter anymore is matter" (p. 801).

Sociomaterialism is a strand of academic work that purposefully theorises materiality. Originating from the field of science and technology studies (STS), sociomaterial perspectives are broadly characterised by a commitment to studying both social and material elements. STS is a relatively new field of academic study that focuses on the nature, practices and impacts of science and technology on society (Bauchspies et al., 2006). Traditionally, STS has focused on how society, as producers and users of technology, is impacted by it. However, as workplaces, schools and other similar institutions became more materially_and in particular, technologically-complex, the more pronounced focus became the need for a perspective that was deliberate in centralising and theorising materiality. While early studies of information and communication technologies often took a rather deterministic point of viewconceptualising the technologies, themselves, as singular drivers of organisational changes - sociomaterial approaches were designed to explore how social and material elements come together to bring about the complexity of society.

Orlikowski, in 2007, and then Orlikowski and Scott, in 2008, began using the term "sociomaterial" as we've come to know it today. They authored some of the seminal contributions in the area and were influenced by Latour's $(1992,2005)$ actor-network 
theory (ANT), Knorr-Cetina's (1997) object-centred sociality, Law's (2004) relational materiality and Barad's (2003) posthumanist performativity.

Ontologically, Orlikowski and Scott's (2008) ideas about sociomaterialism have evolved from earlier STS contributions. Traditionally, STS researchers perceived the world as composed of distinct social and material actors, which hold stable, inherent characteristics. In contrast, Orlikowski and Scott rejected the "dualism" of subject and object, believing instead that social and material actors are, in fact, constitutively entangled-or inseparable.

There continues to be some debate in the realm of sociomateriality, with two primary "camps" being identified: those who consider social (people) and material actors (things) as independent entities pre-existing before they interact and those who believe in the notion of inseparability (Mutch, 2013; Scott \& Orlikowski, 2013). Regardless of the researcher's position amongst these two camps, there is agreement that there are two kinds of agencies: human and material. Perhaps most importantly, the two camps agree that it is within the space that social and material agencies entangle that a more productive discussion can occur.

Sociomateriality, as a concept, has steadily gained momentum. Sociomaterial studies now constitute a significant body of research in STS and in a growing number of academic disciplines, including HPE. For further reading on the tenets and application of sociomaterial perspectives, please refer to Fenwick and Nimmo (2015), MacLeod and Ajjawi (2020) and Nordquist et al. (2020).

\section{Sociomateriality in action}

How do these principles translate into the work we do as sociomaterial researchers in HPE? In the sections that follow, we provide you with a sense of what it means to conduct sociomaterial work, using a case story as an example. Our case focuses on the story of Sam, a chief medical resident (postgraduate medical trainee) feeling a lot of workplace stress. While much of the work that operates from a sociomaterial perspective is focused on technologies and innovation, we specifically elected to demonstrate the power of sociomateriality in action using a problem that is, at first glance, very human-burnout.

In the sections that follow, we'll explore what it means to engage in sociomaterial research, providing more information about how sociomaterial studies feel, look and sound. In each discussion, we'll review some key sociomaterial principles and use Sam's case as an example, to help concretise the discussion.

\section{The case of Sam}

Sam was recently appointed chief medical resident. Sam recognises how privileged it is to be selected for this position-Sam's mentors mention that only the best and brightest are chosen to fulfill this important role. Upon receiving the news, Sam was ecstatic. Having this experience would serve Sam well when it came time for applying to fellowship programs and, eventually, an academic faculty position. Pursuing a career 
in academia had always been Sam's goal. However, these past few months had been difficult. Sam was constantly inundated with tasks and requests. Creating call and clinic schedules, juggling requests for time-off, reviewing and ensuring compliance with hospital policies and professional regulations, leading teaching sessions, supporting the wellbeing and morale of fellow residents, attending department meetings, rounds, journal club, conferences, answering invitations to collaborate on quality improvement projects, the list went on. This was on top of Sam's own clinical and academic activities.

More pressing, the acute care hospital where Sam worked was filled beyond capacity. This was a long-standing issue the hospital faced due to lengthy waits for long-term care spaces and a lack of available home or outpatient care options. Patients were waiting for hours in the emergency department before getting a bed on the ward. The persistent overcrowding left Sam travelling all over the hospital to see patients. Consequently, Sam had little time to establish meaningful rapport with patients or meet face to face with any of the residents on service, let alone grab a nutritious lunch or a few hours of sleep. Communication these days seemed to happen exclusively over email or text. Sam's cellphone had become an additional "appendage", making it difficult to maintain any semblance of home/work life balance.

Until recently, life had been highly focused on training, but now Sam was buckling under the pressure of being an advocate for everyone else. Most of the time, Sam found the role of chief medical resident to be a rewarding experience but lately had begun to disengage from the clinical, academic and administrative activities of the department. Sam has been feeling disillusioned with medicine and thinks burnout might be the culprit. Sam doesn't mention any of this to colleagues or peers for fear of repercussion and judgement.

\section{How do sociomaterial studies feel?}

Sociomaterialism represents an "analytical break" (Contractor et al., 2011) from traditional ways of studying educational issues. Our focus shifts from uniquely social concerns to a more symmetrical analysis, because we believe that the social is "inherently bound up in materiality" (Orlikowski, 2007, p. 1438). These assemblages of social and material elements, then, become the unit of analyses.

It follows, then, that the sociomaterial researcher has a different focus. Rather than paying attention to individual actors - whether social or material — we concentrate on how social and material elements assemble to enact flows of practice and how these practices, in turn, become accepted, or contested, as part of the everyday world (Hultin, 2019). We design studies that allow us to deliberately consider how social and material elements assemble to generate practice. In order to make sense of everyday practice, our work involves "inserting oneself" into the situation in order to better understand the flow of practice (Hultin, 2019, p. 101). Rather than an independent, or detached, observer of a world that is "out there", a sociomaterial researcher conceptualises themself as very much a productive element within the field of analysis. For this reason, reflexivity is particularly important in sociomaterial studies. Given that human-centred methods 
are pervasive in HPE, it can be challenging to maintain the ontological underpinnings of sociomateriality. The sociomaterial researcher must continuously monitor that their study design will allow for actively considering the entanglements of social and material elements.

What does this look like in the real world? Let's return to the case of Sam. How might a sociomaterial researcher "get a feel" for the burnout Sam is experiencing? Rather than focus on Sam as an individual, "burned out" resident, sociomaterialists may begin by problematising the concept of burnout, itself. They might focus on the fact that burnout emerges where multiple practices become entangled and that practices are both materially and socially complex (Hultin, 2019). So, a sociomaterial researcher would most likely begin with a general sense of the problem and design a study that would allow for the researcher to get a sense of the experience of burnout by becoming immersed in the midst of the flow of practice-in our case, this might mean spending time informally observing in the clinical learning environment. The idea would be to explore the entanglements and intricacies of workplace practice that render certain modes of being and acting to be considered desirable and meaningful while others to be less legitimate.

Sam is feeling overwhelmed by the multiple facets of the chief medical resident role. The social expectations, coupled with the educational demands, are high-and the responsibilities are multiple. Sam is juggling curricular requirements, hospital bureaucracy, patient care and a host of other activities. A sociomaterialist might initially document and consider these multiple material elements that seem to be productive with respect to burnout and, then, choose to focus on a particular element of interest. Perhaps the study begins with a suspicion that Sam's ever-present cellphone is an important material or, perhaps, the researcher might choose to focus on the (insufficient) physical space of the hospital Sam seems to be navigating. The next step would be to "zoom in" (Nicolini, 2009) and take a deep dive into how burnout emerges through a network of social and material contributors.

\section{How do sociomaterial studies look?}

Now that we have a "feel" for the work of a sociomaterial researcher, let's focus on what sociomaterial studies look like in action. What types of questions or objectives are best suited to sociomaterial investigations and which methodologies and specific methods fit within a sociomaterial view?

For many sociomaterialists, particularly those who operate from a perspective informed by actor-network theory (ANT), we see our job as opening "black boxes". Latour (1999) described the black box as:

the way scientific and technical work is made invisible by its own success. When a machine runs efficiently, when a matter of fact is settled, one need focus only on its inputs and outputs and not on its internal complexity. (p. 304)

This means that the more familiar and, in turn, taken for granted certain practices become, the less we think critically about how they actually work. 
In order to open black boxes, to get a sense of the complexity of "how things work", sociomaterial empirical work is often ethnographic in nature (MacLeod et al., 2019). While traditional ethnography focuses on understanding culture, sociomaterial approaches focus on understanding the phenomenon under study as a sociomaterial assemblage. This means, we spend time immersed in the field and collect multiple data points in order to attend to both material and social elements. Data collection strategies are layered in order to allow for a depth of analysis and typically include materially oriented variations of 1) artefact and/or document analysis, 2) materially-oriented interviews and 3) observations. For more information on each method, and for an overview of how to engage in sociomaterial ethnography, see MacLeod et al. (2019).

No matter which methods are engaged, the goal of our empirical work as sociomaterialists is to foreground the active nature of materials in influencing or constituting practice. This involves considering material elements symmetrically with social elements. While we use the term "symmetry" to refer to our deliberate efforts to focus on the active nature of materiality, this is not intended to be absurd or unrealistic (at least as such approaches have been used in HPE). Rather, symmetry encourages a focus on the mutual constitution of human and material agency (Parmaggiani \& Mikalsen, 2013), which can be described as entangled or mutually influential. Symmetry, then, refers to the equal theorisation of people, nature and things.

In our case story, Sam, a newly appointed chief medical resident, is overwhelmed by the social and material complexities of the role. The types of research questions we might explore would conceptualise burnout as an assemblage, recognised as an emergent phenomenon that is brought about through an ever-evolving network of social and material elements. These elements include the pride and happiness of being selected chief medical resident and the realities of the role, which include a spectrum of paperwork and tools to manage. So, sociomaterial research questions might attempt to "zoom in" on a particular productive material element, such as "How is the cellphone influencing postgraduate medical education?" Or, the study may "zoom out” (Nicolini, 2009) to look more broadly at systems level considerations, with research questions such as "What factors are contributing to information overload for medical residents?"

Regardless of the question, a sociomaterial study would include multiple methods to allow for in-depth exploration of the complexity of burnout. The study would attempt to account for the fact that the experience itself is tied up in a number of contributing, often invisible, factors that are built into how healthcare professionals fulfill their roles, including for example, patient acuity (high-acuity patients often present challenging medical conditions, influencing wait times in the emergency department, length of stay in hospital, required hospital and outpatient resources); professional guidelines on resident duty hours; the architectural design of the hospital; reliance on asynchronous communication (e.g., email, texting); staff scheduling; documentation requirements; performance metrics; and a myriad of other factors. From this standpoint, the researcher shifts away from analysing the contributors to burnout as part and parcel of medical "culture" or a result of individual deficit (i.e., Sam is not well-suited for the role) to revealing the dynamics that are causing or exacerbating the burnout problem. 
Attuned to the social and material dimensions of burnout, how might we begin to learn more about the complexity of burnout? We may collect documents such as call schedules or terms of reference for the chief medical resident position. We may conduct observations within the hospital where Sam works, taking photographs and field notes to capture the design and layout of the emergency department, ward rooms, the cafeteria and the allocated room where physicians rest while on call. We may interview Sam as well as other chief residents across training programs - not primarily to elicit their perceptions on the prevalence of burnout in medicine but rather to examine how practices become fixed and durable in time and space. The combination of multiple research methods is a deliberate choice on the part of the sociomaterial researcher. The layering of multiple methods brings forward particular insights and understanding, affording a more in-depth and multifaceted examination of the social and material elements of the phenomenon than could be gained from any single method.

\section{How do sociomaterial studies sound?}

Through our discussions of the feel and look of sociomaterial work, a consistent message has been the deliberate foregrounding of materiality. But, for those of us more comfortable with human-centred empirical work, it can be difficult to conceptualise specific strategies sociomaterial researchers can use to hear from silent materials. In other words, how do sociomaterial studies sound?

Not surprisingly, the ways in which a sociomaterial researcher engages in the process of collecting and analysing data will differ radically in contrast to studies that privilege or forefront a more human-centered point of view. As Roehl (2012a) writes, the sociomaterial researcher must "suppress his or her humanist assumption that human beings act and material objects are simply used" (p. 114). This requires vigilance on the part of the researcher, as they must remain attuned to the performativity of material artefacts and re-evaluate their assumed stability and impartiality (Nordstrom, 2018).

Contrary to conventional qualitative research methods that focus largely on generating a deep understanding of another's lived experience as a representational account (e.g., How did that make you feel? What did you learn?), a sociomaterial study is designed to "situate material presence within the realm of qualitative inquiry" (Aagaard \& Matthiesen, 2016, p. 36). To do this effectively means adopting a vocabulary that foregrounds "the sensory and bodily dimensions of practice" (Roehl, 2012b, p. 53). Sociomaterial researchers pose questions deliberately intended to augment the "voice" of materiality (e.g., In what ways does an object invite certain behaviours while inhibiting others? How do materials influence teaching and learning?). Sociomaterial researchers position themselves strategically in the field of study so they can listen, observe and probe about the functionalities, peculiarities, perhaps even the annoyances of working, or having worked with, materials that ordinarily just fade into the background of everyday practice.

In the case of Sam, the sociomaterial researcher may ask to shadow Sam over multiple shifts, respectfully eavesdropping on the conversations taking place in the emergency 
department or between residents in the cafeteria, asking who and what matters in these spaces? Or perhaps the researcher asks Sam to log the usage of mobile technologies over a 24-hour period to explore how materials and discursive practices interact and assemble to generate or intensify feelings of burnout. Keeping this log may then open a space for Sam and the researcher to reflect on the challenges of developing long-lasting relationships with fellow residents or prompt Sam to reveal that the endless stream of emails requiring attention spill into time that would typically be spent with friends and family. Inviting material artefacts, such as the cellphone, into conversation with participants can be a great tool for eliciting stories. From this vantage point, materials can serve as an anchor for retrieving and shaping the stories we tell ourselves and one another (Abidgaard, 2018).

In summary, the feel, look and sound of sociomaterial studies is distinct. While the methods and strategies we use are similar as those used in more familiar, human-centred approaches, sociomaterialists use nuanced versions, designed to highlight the important work of materiality.

\section{Conclusions}

The world of HPE continues to grapple with perennial challenges-issues such as professionalism, assessment, curriculum and, as we raised in this article, burnout. For the most part, the ways in which we study these sticky aspects of HPE have largely focused on the social and human considerations. The insights garnered through humanfocused methods have been immensely meaningful and insightful in our field; however, by singularly focusing on human concerns, we have overlooked the fact that social concerns are intimately entangled with materiality - and that there is potential benefit to studying them as such.

The feel, look and sounds of sociomaterial studies are distinct and require deliberate action on the part of the researcher to foreground material elements. We believe the unique features of sociomaterial projects allow for focused exploration of areas that might otherwise remain silent, or unconsidered. As discussed in the case of Sam, material elements, including physical space, the many technologies involved in education and clinical practice and the multiple forms and documents constituting postgraduate education play a productive role in the very human experience of burnout. By making a deliberate effort to include, and theorise, the work of these non-human elements, new insights may be gleaned.

This paper has outlined what new insights can be unearthed when we make a conscious effort to consider our pervasive HPE challenges and educational circumstances as dynamic, and emergent, interactions between social and material elements. Sociomaterial researchers sensitise us to the amalgam of social and material factors, actively constructing and deconstructing the organisational realities we live, learn and work in, making clear that when we account for materiality, new perspectives and insights may emerge. 


\section{References}

Aagaard, J., \& Matthiesen, N. (2016). Methods of materiality: Participant observation and qualitative research in psychology. Qualitative Research in Psychology, 13(1), 33-46. https://doi.org/10.1080/14780887.2015.1090510

Abildgaard, M. S. (2018). My whole life in telephones: Material artifacts as interview elicitation devices. International Journal of Qualitative Methods, 17, 1-9. https:// doi.org/10.1177/1609406918797795

Barad, K. (2003). Posthumanist performativity: Toward an understanding of how matter comes to matter. Signs, 2(3), 801-831. https:/www.uio.no/studier/emner/ sv/sai/SOSANT4400/v14/pensumliste/barad_posthumanist-performativity.pdf

Bauchspies, J., Croissant, W., \& Restivo, S. (2006). Science, technology and society: A sociological approach. Blackwell.

Contractor, N. S., Monge, P. R., \& Leonardi, P. M. (2011). Multidimensional networks and the dynamics of sociomateriality: Bringing technology inside the network. International Journal of Communication, 5, 682-720. http:// ascnetworksnetwork.org/wp-content/uploads/2010/02/IJoC-Network-Theory2011-ContractorMongeLeonardi.pdf

Faulkner, P., \& Runde, J. (2010). Technological objects, social positions and the transformational model of social activity. MIS Quarterly, 37(3), 803-818. https:// doi.org/10.25300/MISQ/2013/37.3.06

Fenwick, T. (2014). Sociomateriality in medical practice and learning: Attuning to what matters. Medical Education, 48(1), 44-52. https://doi.org/10.1111/ medu. 12295

Fenwick, T., \& Nimmo, G. (2015). Making visible what matters: Sociomaterial approaches for research and practice in healthcare education. In J. A. Cleland \& S. J. Durning (Eds.), Researching medical education (pp. 67-79). John Wiley and Sons.

Hassan, N. R. (2016). Editorial: A brief history of the material in sociomateriality. Data Base for Advances in Information Systems, 47(4), 10-22. https://doi. org/10.1145/3025099.3025101

Hultin, L. (2019). On becoming a sociomaterial researcher: Exploring epistemological practices grounded in a relational, performative ontology. Information and Organization, 29(2), 91-104. https://doi.org/10.1016/j.infoandorg.2019.04.004

Kallinikos, J. (2006). Information out of information: On the self-referential dynamics of information growth. Information Technology and People, 19(1), 98-115. https://doi.org/10.1108/09593840610649989

Knorr-Cetina, K. (1997). Sociality with objects: Social relations in postsocial knowledge societies. Theory, Culture \& Society, 14(4), 1-30. https://doi. org/10.1177/026327697014004001

Latour, B. (1992). Where are the missing masses? Sociology of a few mundane artefacts. In E. Wiebe Bijker \& J. Law (Eds.), Shaping technology/building society: Studies in sociotechnical change (pp. 225-258). MIT Press. 
Latour, B. (1999). Pandora's hope: Essays on the reality of science studies. Harvard University Press.

Latour, B. (2005). Reassembling the social: An introduction to actor-network theory. Oxford University Press.

Law, J. (2004). After method: Mess in social science research. Routledge.

Leonardi, P. M. (2012). Materiality, sociomateriality, and socio-technical systems: What do these terms mean? How are they different? Do we need them? In P. M. Leonardi, B. A. Nardi, \& J. Kallinikos (Eds.), Materiality and organizing: Social interaction in a technological world (pp. 25-48). Oxford University Press.

MacLeod A., Cameron, P., Ajjawi, R., Kits, O., \& Tummons, J. (2019). Actor network theory and ethnography: Sociomaterial approaches to researching medical education. Perspectives on Medical Education, 8(3), 177-186.

MacLeod, A., \& Ajjawi, R. (2020). Thinking sociomaterially: Why matter matters in medical education. Academic Medicine. Advance online publication. https://doi. org/10.1097/ACM.0000000000003143

Mutch, A. (2013). Sociomateriality: Taking the wrong turning? Information and Organization, 23(1), 28-40. https://doi.org/10.1016/j.infoandorg.2013.02.001

Nicolini, D. (2009). Zooming in and out: Studying practices by switching theoretical lenses and trailing connections. Organization Studies, 30(12), 1391-1418. https:// doi.org/10.1177/0170840609349875

Nordstrom, S. N. (2018). Antimethodology: Postqualitative generative conventions. Qualitative Inquiry, 24(3), 215-226. https://doi.org/10.1177/1077800417704469

Nordquist, J., Chan, M. K., Maniate, J., Cook, D., Kelly, C., \& McDougall, A. (2019). Examining the clinical learning environment through the architectural avenue. Medical Teacher, 41(4), 403-407. https://doi.org/10.1080/014215 9X.2019.1566603

Orlikowski, W. J., \& Scott, S. V. (2008). Sociomateriality: Challenging the separation of technology, work and organization. Academy of Management Annals, 2, 433474. https://doi.org/10.1080/19416520802211644

Orlikowski, W. J. (2007). Sociomaterial practices: Exploring technology at work. Organization Studies, 28(9), 1435-1448. https://doi. org/10.1177/0170840607081138

Parmiggiani, E., \& Mikalsen, M. (2013). The facets of sociomateriality: A systematic mapping of emerging concepts and definitions. In M. Aanestad \& T. Bratteteig (Eds.), Nordic contributions in IS research, SCIS 2013: Lecture notes in business information processing (Vol. 156) (pp. 87-103). Springer.

Roehl, T. (2012a). Disassembling the classroom: An ethnographic approach to the materiality of education. Ethnography and Education, 7(1), 109-126. https://doi. org/10.1080/17457823.2012.661591 
A SENSE OF SOCIOMATERIALISM

Roehl, T. (2012b). From witnessing to recording: Material objects and the epistemic configuration of science classes. Pedagogy, Culture and Society, 20(1),49-70. https://doi.org/10.1080/14681366.2012.649415

Scott, S. V., \& Orlikowski, W. J. (2013). Sociomateriality: Taking the wrong turning? A response to Mutch. Information and Organization, 23, 77-80. https://doi. org/10.1016/j.infoandorg.2013.02.003 